\title{
Correction to: Analytic orbit theory with any arbitrary spherical harmonic as the dominant perturbation
}

\author{
Bharat Mahajan $^{1}$ (D) Kyle T. Alfriend ${ }^{1}$ \\ Received: 1 April 2021 / Revised: 1 April 2021 / Accepted: 15 April 2021 / Published online: 20 May 2021 \\ (c) Springer Nature B.V. 2021
}

\section{Correction to: Celestial Mechanics and Dynamical Astronomy (2019) 131:45 https://doi.org/10.1007/s10569-019-9923-3}

In the original paper, the two plots of root-sum-square (RSS) position error of the analytic theories for the orbiter of the Moon and 433 Eros as shown in Figs. 6 and 10 are incorrect. In these plots, the original nondimensional position errors computed through simulation were incorrectly multiplied with the square root of the length unit (the radius of the central body). The corrected versions of Figs. 6 and 10 are provided in this erratum. It is noted that the plots of RSS velocity error in the original figures as well as the errors in the orbital elements of the two orbiters shown in the rest of the figures in the original paper are correct.

The RSS position errors in the corrected figures are greater than that shown in the original paper for the lunar as well as 433 Eros orbiter. In accordance with these corrections, the last sentence of the second-last paragraph in Section 5 of the original paper is modified to reflect the corrected results as "The position and velocity errors of the analytic orbit theory, plotted in Fig. 6, are less than $39 \mathrm{~km}$ and $0.02 \mathrm{~km} / \mathrm{s}$ after 1.6 days of propagation, respectively." Similarly, the last sentence of Section 5 should be read as "The position errors remain below $0.4 \mathrm{~km}$ after nearly 12 days of propagation."

The conclusions of the original paper are not changed in light of the fact that even with the higher position errors (especially in the case of the lunar orbiter), the proposed first-order analytical theory with the first-order secular effects and only zeroth-order long-period effects correctly provides the long-term temporal variations of the orbital elements as shown in Fig. 5 of the original paper.

The original article can be found online at https://doi.org/10.1007/s10569-019-9923-3.

Bharat Mahajan

prince@tamu.edu

1 Department of Aerospace Engineering, Texas A\&M University, College Station, TX 77843-3141, USA 

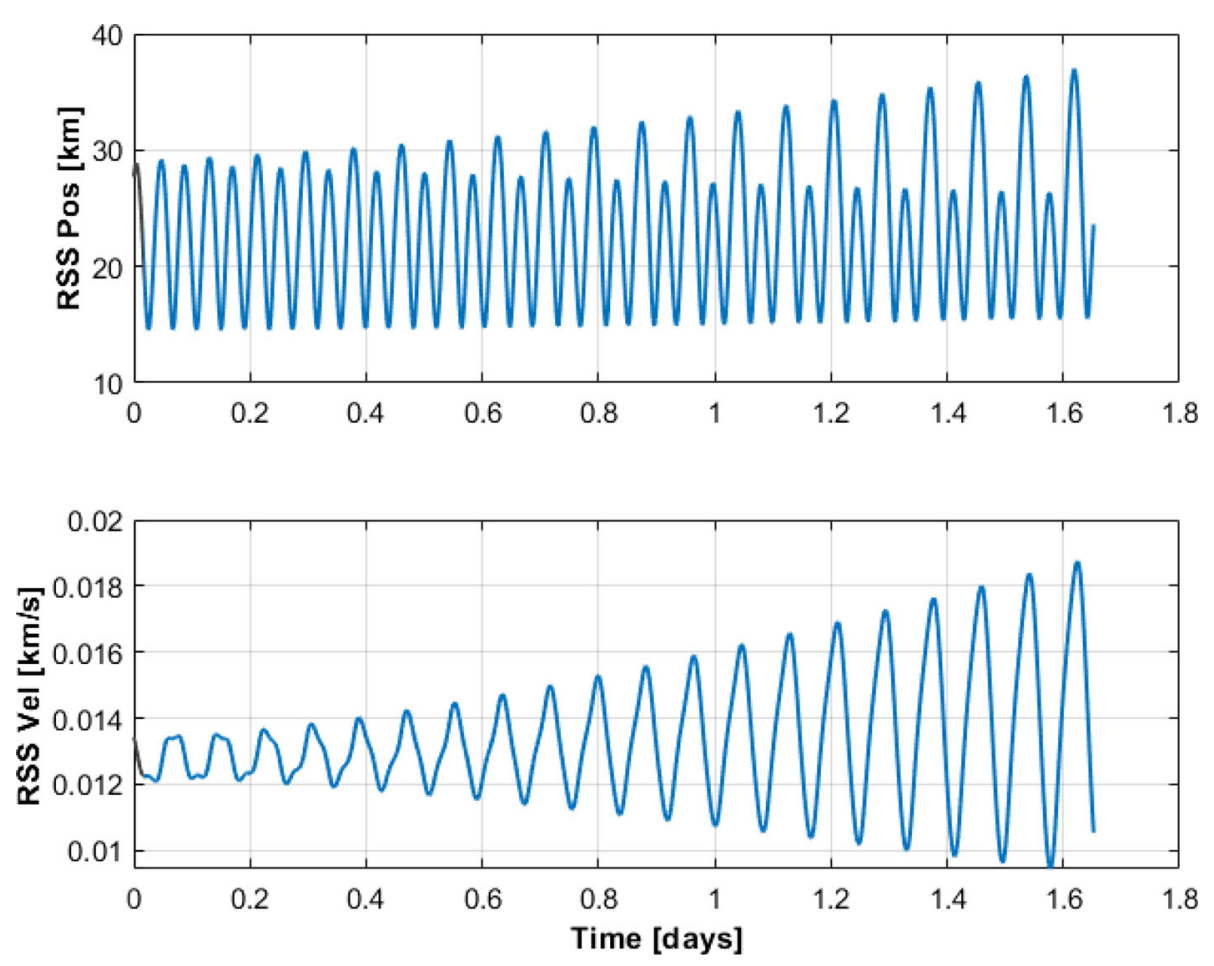

Fig. 6 Root-sum-square position and velocity errors of the analytic orbit theory for the lunar orbiter 

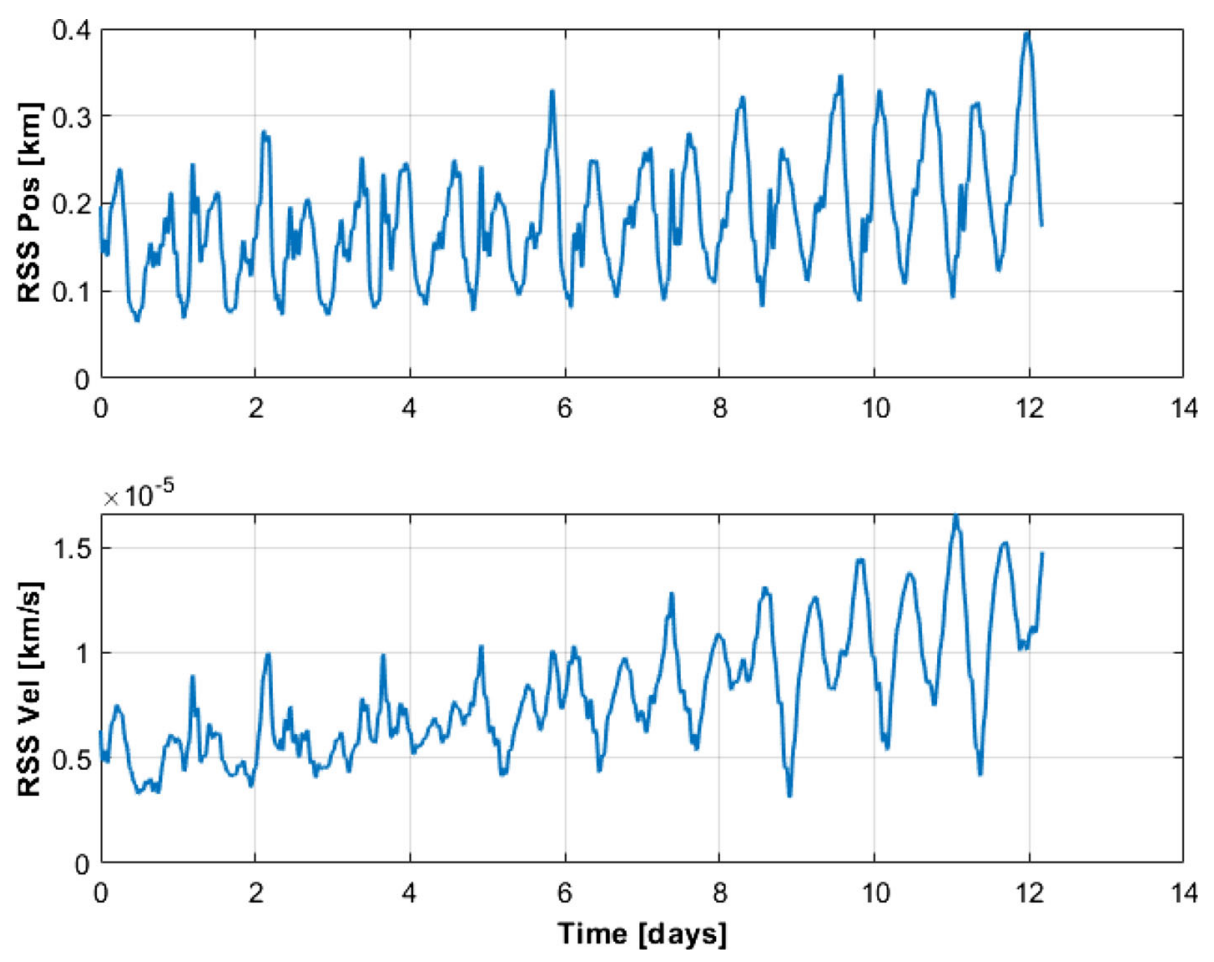

Fig. 10 Root-sum-square position and velocity errors of the analytic orbit theory for the 433 Eros orbiter

Publisher's Note Springer Nature remains neutral with regard to jurisdictional claims in published maps and institutional affiliations. 